\title{
Assessment of Switch Mode Current Sources for Current Fed LED Drivers
}

\author{
Olegs Tetervenoks and Ilya Galkin \\ Riga Technical University, Faculty of Power and Electrical Engineering, \\ Kronvalda Boulevard 1, Riga, Latvia \\ olegs.tetervenoks@rtu.1v, gia@eef.rtu.lv
}

\begin{abstract}
Today solid state lighting is one of the most rapidly growing industries. Unfortunately light-emitting diodes require additional electronics (ballasts, drivers) for proper operation with conventional energy sources. Therefore this paper summarizes the previous studies of LED luminous flux regulation techniques, as well as extends previous studies of current fed (CF) converters. Requirements for the switch mode constant current source are discussed in this paper. Two suitable circuits as well as control algorithm are considered here. PSIM models of the converter were created and the operation with $\mathrm{CF}$ converters was approved. The considerations regarding the practical implementation are given in the end of this paper.
\end{abstract}

Keywords: Light-emitting diode, ballast, LED driver, DC-DC converter.

\section{Introduction}

Today the lighting industry is one of the fastest growing and developing branches in the production of electrical devices. The ground of this is the continued development and increase of efficacy of white light-emitting diodes (LEDs) [1], which are reasonably called the light sources of the future. LEDs gives opportunity to improve lighting system parameters such as efficiency and light quality, therefore also to reduce electrical power consumption. Even greater benefits can be achieved by implementation of smart lighting systems.

\section{Relationship to Collective Awareness Systems}

Today's development should lead to smart lighting systems with self-learning capabilities. In the future LED lighting systems will be equipped with communication modules (such as Bluetooth) for data exchange with personal mobile devices (smartphones) of the user. For the user it will be capable to fit parameters through mobile device using a special application, which also will be connected to a special server. The preferences of the users will be collected and processed on this server and the operation profile for the lighting system can be generated on this server based on the collected data. The data about simultaneous changes of the electricity price and 
availability also can be considered for the generation of profile of lighting system. User will be capable to accept or modify generated profiles and to upload them in lighting system. Therefore such a complex control system interacting with people and taking into account their preferences as well as external factors in future may form a collective awareness lighting system.

Convenient and energy efficient light (luminous flux) regulation is necessary for the implementation of smart lighting systems.

\section{LED Luminous Flux Regulation Methods}

Besides the efficacy of LEDs themselves the performance of the ballast (utilized light regulation technique, efficiency) also plays significant role on the overall efficiency of LED luminaire. Therefore different luminous flux regulation techniques for LEDs and appropriate converters were studied and discussed in previous article [2]. Considered light regulation techniques are summarized in Fig. 1. Their benefits and drawbacks are listed in Table 1. Pulse mode flux regulation is the most appropriate for high performance devices where stable light color temperature is critical (backlit of LCD panels, displays) [3], [4]. This method might suffer from stroboscopic effect (because the luminous flux of LED follows the forward current at very high speed [5]), which is unwanted phenomenon in general lighting. Stroboscopic effect is especially dangerous for industrial lighting, where spinning mechanisms under certain conditions may seem motionless. Therefore step mode and amplitude (fluent) luminous flux regulation methods are the most appropriate in general lighting applications, however, fluent mode regulation technique allows utilizing LED in more efficient way (approximately by $7 \%$ in case of dimming at 50\%) [5].

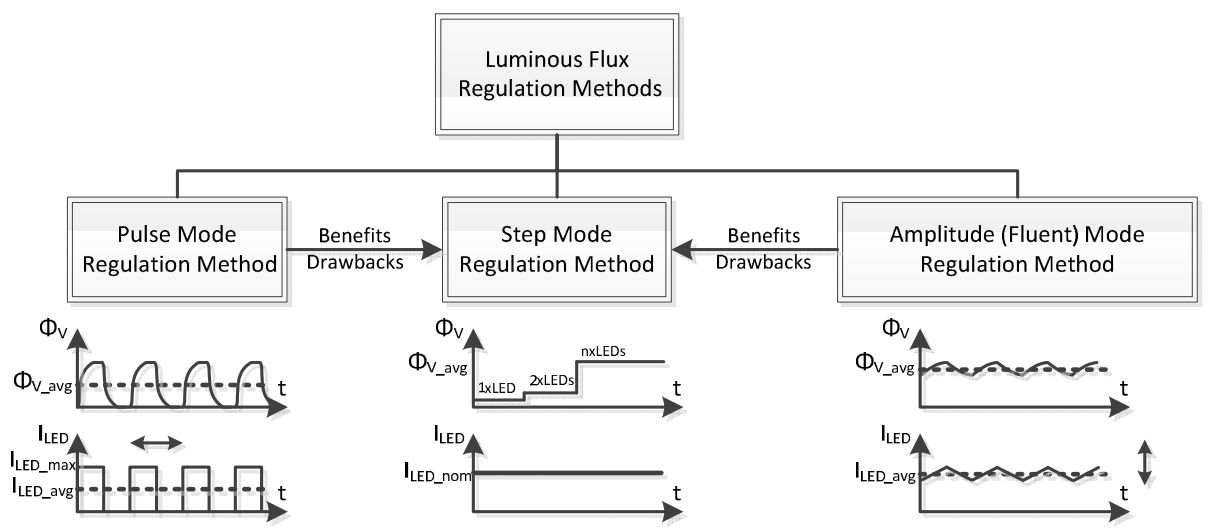

Fig. 1. Luminous flux regulation methods for LED lamps as well as typical waveforms of LED forward current and luminous flux 
Table 1. Benefits and drawbacks of luminous flux regulation methods

\begin{tabular}{|c|c|c|}
\hline Pulse Mode & Step Mode & Amplitude (Fluent) Mode \\
\hline+ high accuracy and resolution & + no stroboscopic effect & + higher efficacy of LEDs \\
\hline + stable color temperature & + stable color temperature & + no stroboscopic effect \\
\hline + simple control system & + simple control system & + longer life span \\
\hline $\begin{array}{l}\text { - undesirable stroboscopic } \\
\text { effect }\end{array}$ & - shorter life span & $\begin{array}{l}\text { - relatively complex control } \\
\text { system }\end{array}$ \\
\hline - shorter life span & $\begin{array}{l}\text { - low resolution (small } \\
\text { number of regulation steps) }\end{array}$ & - unstable color temperature \\
\hline - worse efficacy of LEDs & - worse efficacy of LEDs & $\begin{array}{l}\text { - accuracy and resolution } \\
\text { depends on complexity of } \\
\text { control system }\end{array}$ \\
\hline
\end{tabular}

In Fig. 2 the ampere-lumen (A-Lm) curve of the common high power LED is shown. In case of pulse mode or step mode luminous flux regulation the LED operates at fixed point, usually maximal allowable or nominal (test) current. This maximal or nominal current is applied to LED periodically at high frequency, but the luminous flux is proportional to the duty cycle $\mathrm{D}$ (ratio of the time when the current is applied to the time of whole period.). It can be imagined that change of duty cycle moves the operation point of LED in a straight line which is connected between crossing point of the axes and previously mentioned fixed point (thick dashed lines in Fig. 2).

In case of fluent luminous flux regulation the operation point of LED moves along A-Lm curve in this way achieving higher efficacy, especially at smaller input current (power) as it shown in Fig. 2. Therefore amplitude mode regulation is the most suitable for general lighting applications. A lot of different kind of converters and approaches for amplitude mode regulation were described in scientific papers during last years. This paper deals with current fed $(\mathrm{CF})$ converters.

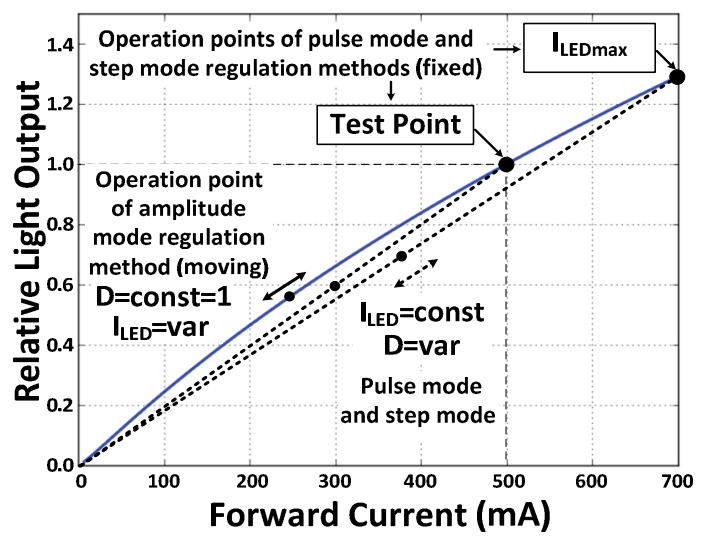

Fig. 2. Ampere-lumen curve of a common high power LED and operation points for different luminous flux regulation methods 


\section{Current Fed LED Drivers}

LEDs are current consumers rather than voltage consumers. At the same time the most of the today's LED drivers are based on voltage fed (VF) converters, which means LED current (luminous flux) is controlled indirectly through applied voltage (volt-ampere V-A stage in Fig. 3). In previous studies it has been hypothesized that current fed $(\mathrm{CF})$ converters are more suitable for driving LEDs, as the LED current is regulated in direct way [6], [7]. Also the circuits for three basic topologies of the current fed converters have been derived [7]. CF Buck topology (Fig. 4 a) is the most appropriate for step down applications. Also it has convenient controllability curve (Fig. 4 b).

$\mathrm{CF}$ drivers require constant current source at the input (Fig. 4). Current sources based on transistors operating in active region are energy inefficient, therefore the possibilities to build switch mode constant current source are considered in the following section.

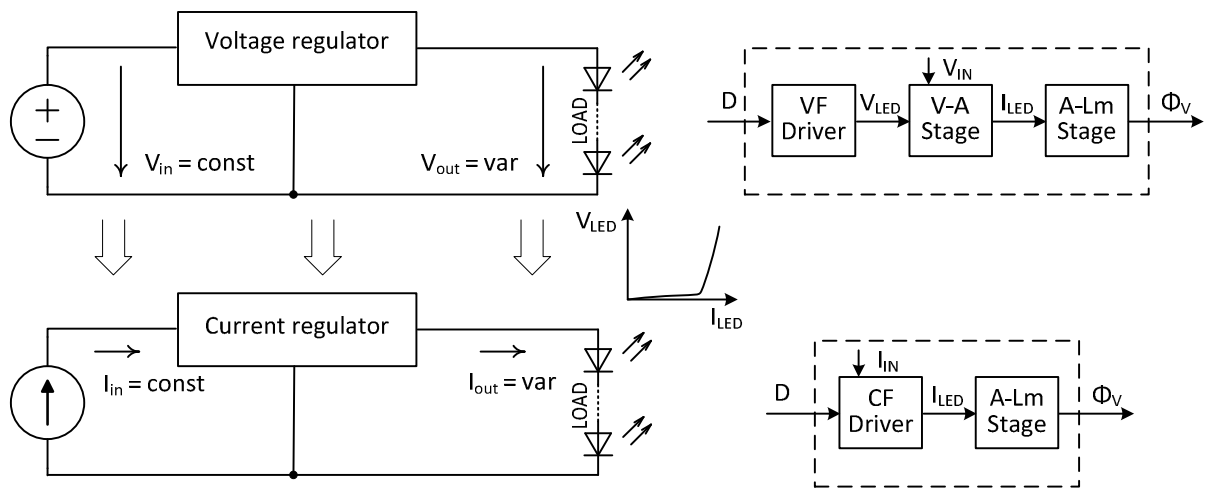

Fig. 3. Transition from voltage fed to current fed converters. Functional diagram of the light regulation loops for VF and $\mathrm{CF}$ chains.

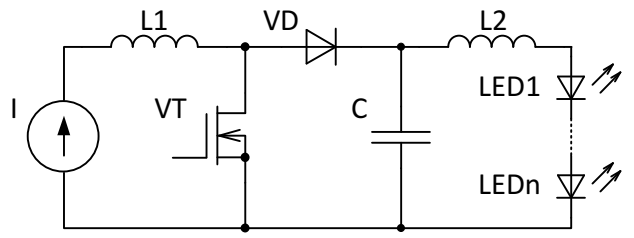

a)

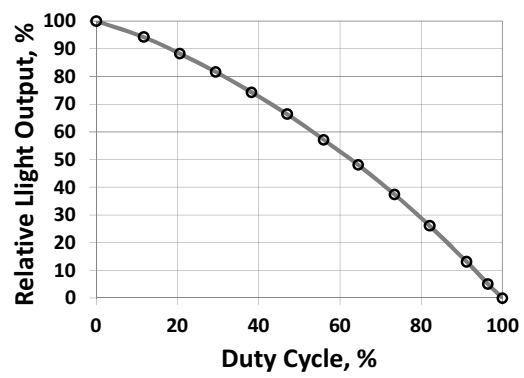

b)

Fig. 4. CF buck converter: a) electrical principal circuit; b) controllability curve [6] 


\section{$5 \quad$ Switch Mode Constant Current Sources}

The primary energy source almost always is a voltage source. This means constant current source must be voltage fed converter operating in constant output current mode. The requirements for the constant current source are:

1. uninterruptible current flow at the output

2. the capacitors (voltage source) at the output are not allowed in order to prevent high short circuit current.

The inductor is the only element, which meet the first requirement. Therefore the inductor current must be input current of the CF driver (inductor must be connected in series with the input of CF driver).

In [8] non-inverting buck-boost converter is described (at least two controllable switches). In fact it is combination of VF buck converter, which operates as constant current source and $\mathrm{CF}$ buck converter, which operates as current regulator. The constant current is formed in inductor, which is between VF and CF parts of the converter. In [8] it has been hypothesized that constant current in this inductor can be achieved by synchronized operation of the switches. The main feature of control law for the switches in this case: the sum of duty cycles of both switches must be approximately $100 \%$ [8]. Non-inverting buck-boost converter is capable to provide output voltage less or equal to the input voltage, but the efficiency decreases at higher difference between input and output voltage. It becomes a serious problem at high input voltage (rectified mains voltage). Also galvanic isolation is necessary for safety reasons. Therefore the converter with pulse transformer is necessary.

Special attention should be paid to the possibility of saturation of the core. Transformer isolated converter with bidirectional flow of current in primary winding should be used to prevent this. The circuit shown in Fig. 5 is based on half-bridge converter. It has been hypothesized that the similar control law as in [8] can be used for circuit shown in Fig. 5. For half-bridge converter the maximal allowable duty cycle of switches VT1 and VT2 is $50 \%$, then control law for this circuit can be written as:

$$
D_{V T 1}+D_{V T 2}+D_{V T 3}=2 \cdot D_{V T 1}+D_{V T 3}=100 \% \text {. }
$$

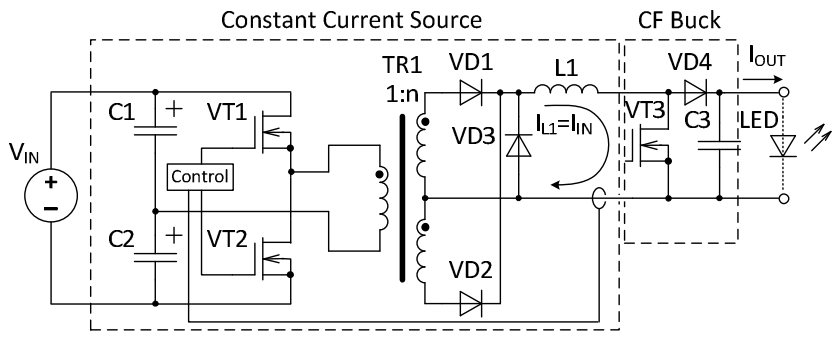

a)

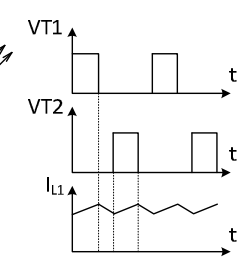

b)

Fig. 5. Constant current source based on half-bridge converter: a) configuration; b) typical waveforms 


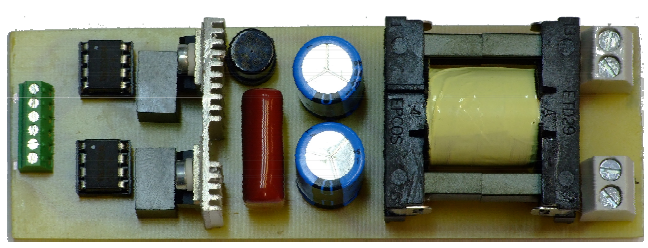

a)

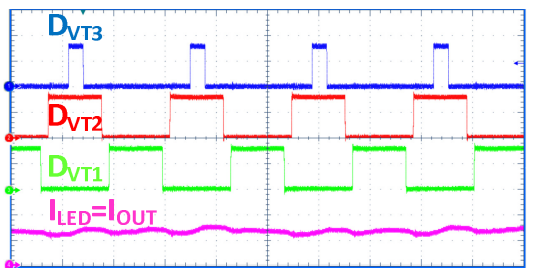

b)

Fig. 6. The prototype of half-bridge based constant current source for CF buck converter: a) photo of the prototype; b) typical control signals $\left(\mathrm{D}_{\mathrm{VT} 3} \approx 12 \%\right)$ and the waveform of the output current

Table 2. Results of the experiments with the prototype shown in Fig. 6 (at constant input voltage $260 \mathrm{~V}$ and 7 connected in series high power LEDs as a load)

\begin{tabular}{c|c|c|c}
\hline $\mathrm{D}_{\mathrm{VT} 3}, \%$ & $\mathrm{P}_{\mathrm{IN}}, \mathrm{W}$ & $\mathrm{P}_{\mathrm{OUT}}, \%$ & Efficiency, $\%$ \\
\hline 12.1 & 29.9 & 27.5 & 91.9 \\
\hline 49.8 & 6.4 & 5.4 & 85.2 \\
\hline $\mathbf{8 7 . 4}$ & 2.6 & 1.7 & 63.7 \\
\hline
\end{tabular}

But the relationship between the input voltage $V_{\text {IN }}$, output voltage $V_{\text {OUT }}$, duty cycle $D$ and transformer turns ratio $\mathrm{n}$ is given in the following expression

$$
V_{\text {OUT }}=0.5 \cdot n \cdot D \cdot V_{I N} \text { or } D=\frac{2 \cdot V_{\text {OUT }}}{n \cdot V_{I N}} \text {. }
$$

The prototype shown in Fig. 6 was built to verify hypothesis (1) and the results of several experiments are summarized in Table 2. The conclusion from these preliminary experiments may be the same as in [8]: the duty cycle itself $\left(\mathrm{D}_{\mathrm{VT} 3}\right)$ is the main regulation parameter while the balance (1) plays additional tuning role. Synchronized operation of the switches is not enough to ensure stable operation of the whole system: closed loop regulation is necessary.

\section{Considerations about the Control System}

The most critical node of considered circuits is the control system. Functional diagram of control loop is shown in Fig. 7. In this control loop the output current set point value is compared with the actual output current value. The difference of them is the error, which increases or decreases initial duty cycle to adjust output current. Initial duty cycle must be calculated simultaneously (from (2)) using input and output voltage of the converter (feedback and feedforward loops). In simplified version it can be assumed that the input voltage is constant (two feedback loops). Therefore only the value of output voltage can be used for initial duty cycle calculations (Fig. 7).

To verify the functionality the model was simulated in PSIM software. PSIM model with control system discussed above is shown in Fig. 8. 


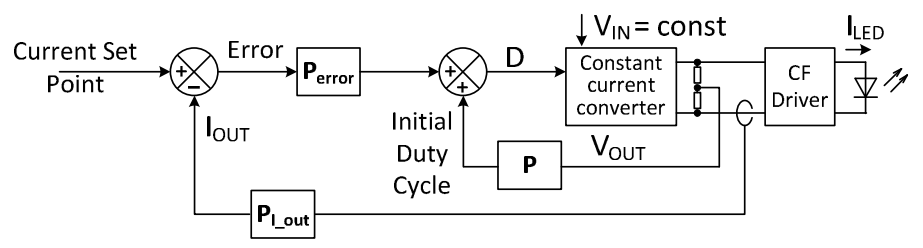

Fig. 7. Functional diagram of control loop for considered constant current converter

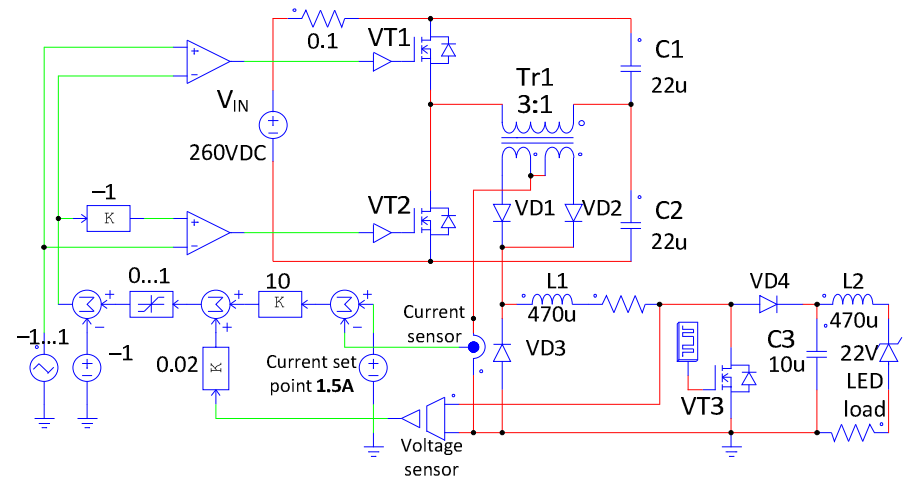

Fig. 8. PSIM model for considered constant current converter

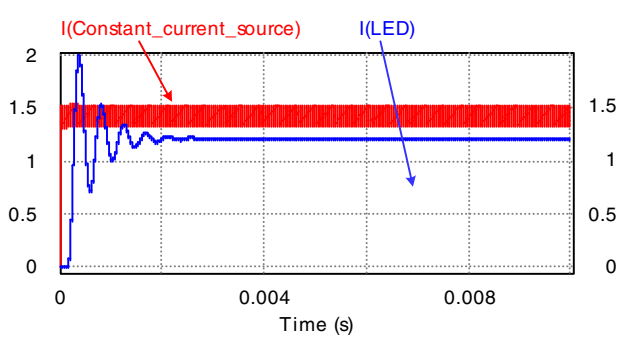

a)

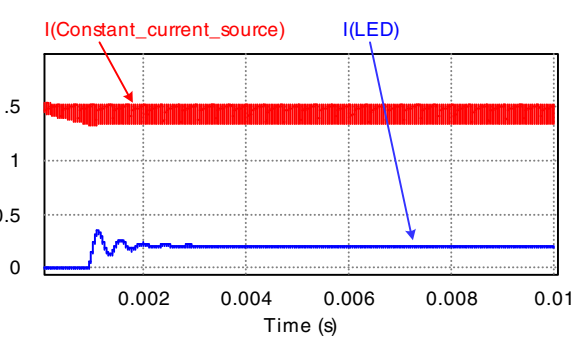

b)

Fig. 9. Simulation results of the considered constant current sources operating with $\mathrm{CF}$ buck converter: a) startup process at $85 \%$ of maximal LED current ( $D=15 \%$ for CF buck); b) steady state operation at $15 \%$ of maximal LED current ( $D=85 \%$ for $C F$ buck).

The simulation results for the considered circuit operating with the $\mathrm{CF}$ buck are shown in Fig. 9. It should be noted that this model operate switches of the constant current source and CF buck asynchronously. The simulation results Fig. 9 show that this configuration of control system is capable to operate properly. 


\section{$7 \quad$ Summary and Conclusions}

Smart lighting systems require convenient and energy efficient approaches for the regulation of light level. Current fed drivers are capable to meet these requirements, but a constant current source is necessary at the input for their proper operation.

The possibilities to build constant current source for the $\mathrm{CF}$ driver have been discussed in this paper. Half-bridge based constant current source prototype was built and experimentally verified for synchronized operation. It was found that additional closed loop regulation of the system is necessary for stable operation. Asynchronous operation with closed loop regulation was verified using PSIM model.

The practical implementation of the CF LED drivers with constant current sources described in this paper is the task for the further work. The combination of synchronous operation of both parts of the converter and the closed loop regulation is the topic for further research.

Acknowledgment. This work has been supported by the European Social Fund within the project «Support for the implementation of doctoral studies at Riga Technical University».

\section{References}

1. Cree, Cree Reaches LED Industry Milestone with 200 Lumen-Per-Watt LED, Press Releases (December 18, 2012), http://www.cree.com/news-and-events / cree-news / press-releases 2012 / december/mkr-intro

2. Milaševski, I., Tetervenoks, O., Galkin, I.: Assessment of Energy Efficient LED Ballasts Based on their Weight and Size. Power and Electrical Engineering 29, 105-112 (2011) ISSN 1407-7345, doi:10.2478/v10144-011-0018-6

3. Chen, W., Hui, S.Y.R.: A Dimmable Light-Emitting Diode (LED) Driver With Mag-Amp Postregulators for Multistring Applications. IEEE Transactions on Power Electronics 26(6), 1714-1722 (2011), doi:10.1109/TPEL.2010.2082565

4. Chiu, C.-L., Chen, K.-H.: A high accuracy current-balanced control technique for LED backlight. In: IEEE Power Electronics Specialists Conference, PESC 2008, June 15-19, pp. 4202-4206 (2008), doi:10.1109/PESC.2008.4592615

5. Schmid, M., Kuebrich, D., Weiland, M., Duerbaum, T.: Evaluation on the Efficiency of Power LEDs Driven with Currents Typical to Switch Mode Power Supplies. In: 42nd IAS Annual Meeting Industry Applications Conference. Conference Record of the 2007 IEEE, September 23-27, pp. 1135-1140 (2007), doi:10.1109/07IAS.2007.178

6. Milashevski, I., Galkin, I., Tetervenok, O.: Assessment of buck converter powered by current or voltage sources for LEDs luminary. In: 2012 13th Biennial Baltic Electronics Conference (BEC), October 3-5, pp. 239-242 (2012), doi:10.1109/BEC.2012.6376861

7. Galkin, I., Tetervjonok, O., Milashevski, I.: Comparative study of steady-state performance of voltage and current fed dimmable LED drivers. In: 2013 8th International Conference on Compatibility and Power Electronics (CPE), June 5-7, pp. 292-297 (2013), doi:10.1109/CPE.2013.6601172

8. Galkin, I., Tetervenoks, O.: Validation of Direct Current Control in LED Lamp with NonInverting Buck-Boost Converter. In: 2013 39th Annual Conference of the IEEE Industrial Electronics Society (IECON), November 10-13, pp. 6019-6024 (2013) ISBN 9781479902231 